\title{
Research on the Presentation and Development of Digital Media Art in an Open Environment
}

\author{
Zhang Yanhua \\ Shandong Xiehe Univerisity, Shandong, China, 250100
}

Keywords: digital media art; open environment; development; presentation characteristics

Abstract: With the continuous development of information technology and computer technology, people have gradually entered the digital information age, which has also prompted many industries to have undergone radical changes. In the open environment, digital media art has a new development and different characteristics. Digital media art is a brand new subject that integrates art and digital technology. It gathers with other art disciplines and has significant epochal and interdisciplinary characteristics. Therefore, the study of digital media art needs to be placed in the current environment to better understand it. In this regard, this paper introduces the characteristics of digital media art in the current open environment, and analyzes in detail the development trend of digital media art in an open environment.

\section{Introduction}

With the advent of the digital information age, digital media art has a new space for communication and publicity in this open environment, and its application in packaging, advertising, drawing, design and other aspects is more significant. Promote the development of digital media art in the long run. For digital media art, it is a new form of art. Its composition is shown in Figure 1. It requires a multi-disciplinary and multi-knowledge level to complement each other. Therefore, we must learn to use digital technology to combine digital technology with artistic creation to create a true digital media art, so that we can continue to develop and innovate digital media art.

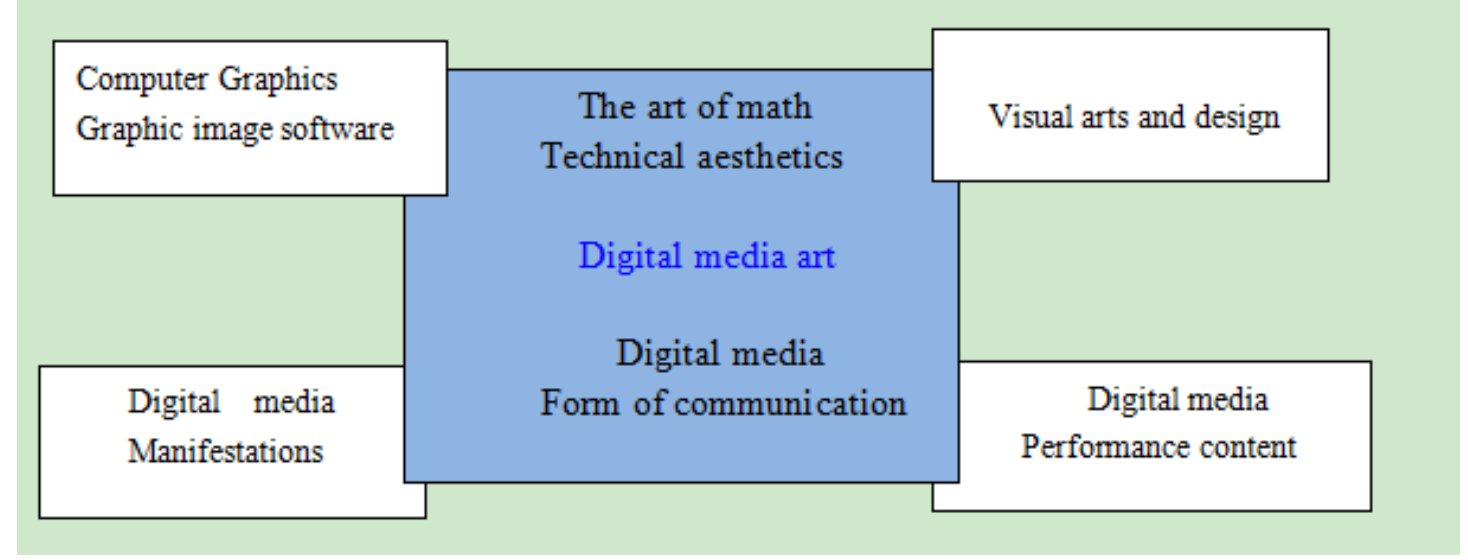

Figure 1 Graphical composition of digital media art 


\section{The characteristics of digital media art in an open environment}

\subsection{Network technology}

The characteristics of the era of digital media art are not found in classic art works, which is also in line with the development needs of the times. In a highly information-based, networked society, these two characteristics of art works are a product of the development of the times and a manifestation of the flexible use of digital media art. The addition of computer network technology makes digital media art works more diversified, enriched and visualized. At the same time, combining the cultural knowledge of multiple disciplines, the art works are more full of depth and conducive to the performance of the works ${ }^{[1]}$.

\subsection{Popularity}

With the improvement of people's material quality of life, the demand for spiritual life also increases. Digital media technology is a combination of science and technology and art. It can be said that it is an artistic product with a modern flavor, which meets the development needs of The Times and also meets the needs of people's spiritual life. On this basis, as the universality of digital media technology affects the development of every relevant technology, digital media works of art also have a strong universality and dissemination force, which can be highly recognized at every different level in the social environment.

\subsection{Space construction}

Many famous art works pay special attention to the treatment of exhibition space in the display process, because the charm of artistic creation not only comes from the work itself, but also is affected by the space in which the art works are located. The treatment of space can reveal the cultural connotation of some art works. Digital media art works have the unparalleled characteristics of "virtual space construction" because of its related technology, and it has unique advantages in space construction. Whether it is to solve the problem of spatial distance or to express the cultural connotation of the work by changing the space, this is all that digital media art can easily do. This is the embodiment of the combination of digital media technology in the development of art creation and artistic expression. And gradually developed into a unique artistic charm of digital media art works.

\subsection{Communication}

In the past, when computer network technology was not very developed, the dissemination of art works could only rely on the simple display of works. The intensity and efficiency of communication were far less than now. Many excellent literary and artistic works were not appreciated, and many excellent literary creators. Unrecognized digital media technology is the product of advanced computer and network technology, and natural digital media art works are also characterized by the convenience of network technology. Not only can artistic works be presented to the audience in the first place, but also the communication between the audience and the creators. The audience can send emails or messages through the Internet, and the creators can share the personal understanding of the works instantly, which is very convenient and fast. 


\subsection{Virtualization}

Classic art works are physically manifested in physical form, and people can intuitively appreciate art works during the exhibition. In digital media art, the development of digital technology and image technology allows art works to appear on computers and spread on the Internet. As shown in figure 1, the market prospects of Chinese classic art works are getting better and better after using digital media technology. The virtualization of artistic works is a great progress in cultural communication, and also a manifestation of the characteristics of the development of the times. It is conducive to the development and dissemination of excellent works of art, and provides a convenient channel for audiences who are keen to appreciate art works. Digital media technology is like a tool in the hands of art creators. The unprecedented form of expression allows art works to be displayed in the same way, both technological advancement and cultural sublimation.

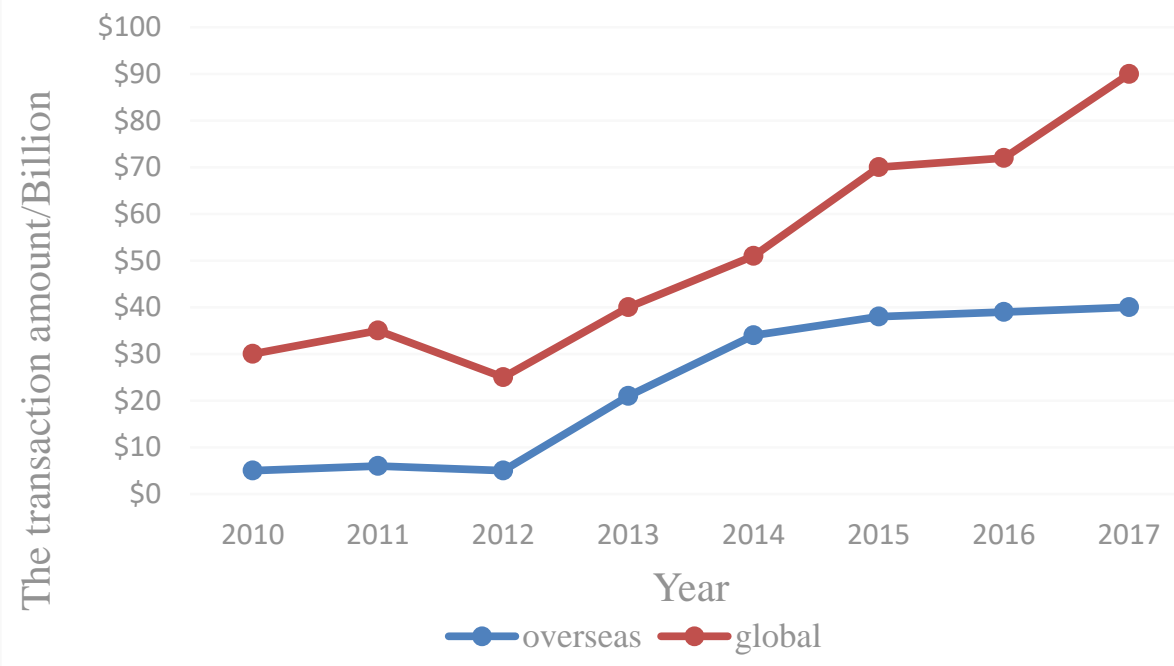

Figure 1 Market sales of Chinese classic art works after using digital media technology

\section{The development of digital media art in an open environment}

\subsection{Promote a broader design space}

With the ability of digital technology, digital media art can continuously innovate the organization, work form, performance method, design efficiency and design concept of art design, and promote the digital media art to have a new look and form a significant shocking effect.

In the digital society, the influence of digital media art on design objects and the environment is obvious. The application of a large number of modern technologies can design design effects that cannot be realized by traditional art design. At the same time, digital media art design relies on the experience of traditional art design, constantly references and innovates the traditional art design factors, thus designing more intelligent colors, making the design products more humanized, making the design content more vivid, and letting the technology environment more enthusiastic, no longer indifferent. In addition, under the support of the open environment, the design content of digital media art can be spread and publicized in a more diversified way, which also promotes a broader design approach, and the design is no longer limited to rigid images and objects. It can also be a three-dimensional experiential service. 


\subsection{Promote richer design content}

The form and medium of communication that new media possess can provide an effective improvement and promotion for conveying artistic design content. Digital TV, interactive media, interactive games, networks and virtual spaces form the main medium and form of information dissemination. The traditional visual communication design range can achieve innovative breakthroughs. The digital carrier can significantly enhance the content and design of visual communication. Digital media art design inherits traditional art design in aesthetic assessment and theoretical design. Digital media art design is a derivative of modern design development. It has a certain era, and the amount of information and performance is also very rich, with a broader era and science $^{[4]}$.

\subsection{Promote more diversified design methods}

In traditional art design, designers often rely on manual painting to build stereograms, plans, sketches, ceiling plans and renderings, but the design and manufacture are not done by the same person. After the product is finished, the designer will follow the product. Static effects or static graphs to analyze and optimize the effects of the design. However, the user is a dynamic product, which will experience the product from multiple levels and form an interactive experience with the product. This is obviously different from the connection between the designer and the product. The designer also needs to use the user. The feedback can better grasp the effect of the design, which also leads to the vacuum between design and use.

In the digital media art design under the open environment, digital technology can better show the identity between the designer and the user, and realize the characteristics of more stable design, easier modification and more convenient storage. The vacuum between design and use, digital art design allows designers to complete the design work in real use scenarios, thereby improving the overall effectiveness of the design, and avoiding the various constraints of traditional design in certain aspects. Promote coherence in design, manufacturing, use and conception, and promote products to be more visual and humanized.

\section{Conclusions}

Today's society is a highly information-based and digital era. All walks of life pursue informatization and digitalization in business, and digital media art is the product that meets the development needs of the industry. Digital media art continues to develop and innovate, and its features are more distinct. The integration of technology and art has been promoted significantly in an open environment. The use of digital technology can provide more rich artistic design styles and methods for modern art design. At the same time, the development and innovation of modern design art have also significantly improved the practicality and usability of digital media technology. It can be seen that the development of digital media art in an open environment is interactive, and the two can achieve mutually promoting development and innovation.

\section{References}

[1] Zhang Yating. The ecosystem of digital media art under open data resources [D]. Lu Xun Academy of Fine Arts, 2016.

[2] Xu Shengchao. Analysis of Visual Recognition Based on Digital Media Art Environment [J]. Beauty and the Times (I), 2013(11): 68-70.

[3] Hu Bo. Exploring the Application of Reverse Thinking in Digital Media Art [J]. Shandong Industrial Technology, 2017(4): 146-147. 
[4] Xu Liwei. New Features of Digital Media Art in Display Design[J]. Modern Decoration(Theory), 2013(10):8-8.

[5] Hu Yan. Research on the Development Strategy of Digital Media Art Industry in China[J]. Journal of Nanjing University of Finance and Economics, 2014(4): 101-104. 